\title{
A combination of the immunohistochemical markers CK7 and SATB2 is highly sensitive and specific for distinguishing primary ovarian mucinous tumors from colorectal and appendiceal metastases
}

\author{
Nicola S. Meagher $\mathbb{1}^{1,2,3}$ et al. \\ Received: 18 March 2019 / Revised: 17 May 2019 / Accepted: 18 May 2019 / Published online: 25 June 2019 \\ (c) The Author(s), under exclusive licensc to United States \& Canadian Academy of Pathology 2019
}

\begin{abstract}
Primary ovarian mucinous tumors can be difficult to distinguish from metastatic gastrointestinal neoplasms by histology alone. The expected immunoprofile of a suspected metastatic lower gastrointestinal tumor is $\mathrm{CK} 7^{-} / \mathrm{CK} 20^{+} / \mathrm{CDX} 2^{+} / \mathrm{PAX} 8^{-}$. This study assesses the addition of a novel marker SATB2, to improve the diagnostic algorithm. A test cohort included 155 ovarian mucinous tumors (105 carcinomas and 50 borderline tumors) and 230 primary lower gastrointestinal neoplasms (123 colorectal adenocarcinomas and 107 appendiceal neoplasms). All cases were assessed for SATB2, PAX8 CK7, CK20, and CDX2 expression on tissue microarrays. Expression was scored in a 3-tier system as absent, focal (1-50\% of tumor cells) and diffuse ( $>50 \%$ of tumor cells) and then categorized into either absent/present or nondiffuse/diffuse. SATB2 and PAX8 expression was further evaluated in ovarian tumors from an international cohort of 2876 patients (expansion cohort, including 159 mucinous carcinomas and 46 borderline mucinous tumors). The highest accuracy of an individual marker in distinguishing lower gastrointestinal from ovarian mucinous tumors was CK7 (91.7\%, nondiffuse/diffuse cut-off) followed by SATB2 (88.8\%, present/absent cut-off). The most effective combination was CK7 and SATB2 with accuracy of 95.3\% using the 3-tier interpretation, absent/focal/diffuse. This combination outperformed the standard clinical set of CK7, CK20 and CDX2 (87.5\%). Re-evaluation of outlier cases confirmed ovarian origin for all but one case. The accuracy of SATB2 was confirmed in the expansion cohort (91.5\%). SATB2 expression was also detected in $15 \%$ of ovarian endometrioid carcinoma but less than 5\% of other ovarian histotypes. A simple two marker combination of CK7 and SATB2 can distinguish lower gastrointestinal from ovarian primary mucinous tumors with greater than $95 \%$ accuracy. PAX8 and CDX2 have value as second-line markers. The utility of CK20 in this setting is low and this warrants replacement of this marker with SATB2 in clinical practice.
\end{abstract}

\section{Introduction}

Primary gastrointestinal neoplasms can present as metastatic ovarian masses and their potential to mimic an ovarian primary neoplasm, mostly mucinous type, is well recognized [1-4]. Ancillary immunohistochemistry is often applied with the standard panel including CK7, CK20 and CDX2. The expected immunoprofile of a lower gastrointestinal tumor is CK7 negative, CK20 positive and CDX2 positive, with the reverse generally associated with a primary ovarian tumor. The clinical utility of this profile is

Supplementary information The online version of this article (https:// doi.org/10.1038/s41379-019-0302-0) contains supplementary material, which is available to authorized users.

Extended author information available on the last page of the article hampered by reduced specificity due to focal and even diffuse positivity of CK20 and CDX2 in mucinous ovarian tumors [4]. This limitation warrants additional studies to validate more specific markers such as SATB2 and PAX8 [5-7]. SATB2 (special AT-rich sequence-binding protein 2) is a transcriptional regulator (encoded on chromosome 2q32-33), that is involved in osteoblastic and cortical neuron differentiation, and in skeletal development [8]. SATB2 is also expressed in epithelial cells of the lower gastrointestinal tract including the colon and appendix, therefore is expected to be present in lower gastrointestinal tumors, but not primary ovarian neoplasms [9]. Another transcription factor, PAX8, is highly expressed in müllerian epithelium (including approximately half of mucinous ovarian tumors), kidney and thyroid but not in lower gastrointestinal tumors [10]. 
Among the five main histotypes of ovarian carcinoma, mucinous carcinoma is the least common, accounting for only $3-4 \%$ of cases. This proportion is significantly lower than earlier estimations of $\sim 12 \%$ [11]. The difference is likely due to improved recognition of metastatic adenocarcinomas to the ovary that mimic primary ovarian mucinous tumors. Despite these improvements, accurate diagnosis remains a challenge in clinical practice, with a lack of standardization in testing, and uncertainty over optimum cut-offs with respect to focal and diffuse staining of immunohistochemical markers [12]. Due to its rarity, it is challenging to accumulate sufficient cases of mucinous carcinomas in a research setting to investigate this poorly understood histotype [13]. Previous studies of the Ovarian Tumor Tissue Analysis (OTTA) consortium included 6-7\% of mucinous carcinomas [14-17].

The primary aim of this study was to compare the sensitivity, specificity and accuracy of CK7, CK20, CDX2, SATB2, and PAX8 expression individually, and in combination to identify the most efficient panel to differentiate primary ovarian mucinous neoplasms (herein ovarian mucinous tumors refer to atypical proliferative/mucinous borderline tumors and mucinous carcinomas) from lower gastrointestinal primaries (colorectal adenocarcinomas and appendiceal neoplasms) in a well characterized test cohort. A second objective was to validate the specificity of SATB2 in a large expansion OTTA cohort including all the main ovarian carcinoma histotypes and explore survival associations of SATB2 and PAX8 in mucinous carcinomas.

\section{Materials and methods}

\section{Study population - test and expansion cohorts}

Cases for the test cohort were ascertained as a subset of the Ovarian Tumor Tissue Analysis (OTTA) Consortium with paraffin-embedded tissue available for staining. The test cohort underwent contemporary histological review with integration of immunohistochemical markers to assure accurate histotyping [18]. During this process, mucinous cases were also assessed for features of metastatic adenocarcinoma. These features were surface or hilar involvement by carcinoma, nodular pattern, destructive invasion, single cells, or signet ring cells [1]. Features suggestive of involvement by low-grade appendiceal neoplasms were subepithelial clefts, scalloped glands, pseudomyxoma ovarii, tall hypermucinous cells, fibrous hypocellular stroma and absence of mucin granulomas [2]. Matched clinical data were available (Supplementary Table S1), and all cases were stained and scored for all 5 immunohistochemical markers. A cohort of 123 primary colorectal adenocarcinomas (all Stage II) was investigated for comparison, as well as 107 appendiceal neoplasms, which have previously been described [18].

The expansion cohort $(n=2876$ cases of the major ovarian histotypes) was drawn from 14 centers participating in the OTTA consortium, with the initial diagnosis classified according to the original pathology report or following specialized central review [19] (Supplementary Table S2). These cases were all scored for SATB2 and PAX8.

\section{Immunohistochemistry and scoring}

All staining was performed in a central pathology laboratory. Samples were assembled in tissue microarrays, with duplicate or triplicate cores. Immunohistochemistry was performed on $4-\mu \mathrm{m}$ sections from tissue microarrays on a DAKO Omnis platform. Immunohistochemical staining method details are provided in Table 1. Two SATB2 antibodies were used for both the lower gastrointestinal and ovarian cohorts, and the concordance between the two was assessed (Supplementary Table S3).

All markers were scored in a 3-tier system as absent if tumor cells showed no staining, focal if $1-50 \%$ of tumor cells exhibited unequivocal staining or as diffuse if $>50 \%$ of tumor cells were stained in their respective subcellular compartment (nuclear for SATB2, PAX8, CDX2, cytoplasmic for CK7 and CK20), (Fig. 1). The staining intensity of SATB2 was generally strong with only occasional cases showing weak intensity, which might be related to tissue antigenicity.

Table 1 Immunohistochemical staining details

\begin{tabular}{|c|c|c|c|c|c|c|}
\hline Marker & Supplier & Clone & Antigen retrieval & $\begin{array}{l}\text { Primary } \\
\text { dilution }\end{array}$ & Incubation & Platform \\
\hline SATB2 (1) & Abcam & SATBA4B10 & 30 min HIER using high $\mathrm{pH}$ Tris buffer & $1: 400$ & $30-10 \mathrm{M}-30$ & DAKO Omnis \\
\hline SATB2 $(2)^{*}$ & Abcam & EPNCIR130A & 30 min HIER using high $\mathrm{pH}$ Tris buffer & $1: 800$ & $30-10 R-30$ & DAKO Omnis \\
\hline PAX8 & Cell Marque & MRQ-50 & 30 min HIER using high $\mathrm{pH}$ Tris buffer & $1: 200$ & $30-10 R-30$ & DAKO Omnis \\
\hline CK7 & Dako & OV-TL 12/30 & 30 min HIER using high $\mathrm{pH}$ Tris buffer & RTU & $12 \times 20$ & DAKO Omnis \\
\hline CK20 & Dako & KS20.8 & 30 min HIER using high $\mathrm{pH}$ Tris buffer & RTU & $25 \times 20$ & DAKO Omnis \\
\hline $\mathrm{CDX} 2$ & Dako & DAK-CDX2 & 30 min HIER using high $\mathrm{pH}$ Tris buffer & RTU & H10-10M-10 & DAKO Omnis \\
\hline
\end{tabular}

$R T U$ ready to use; *used in main analyses 


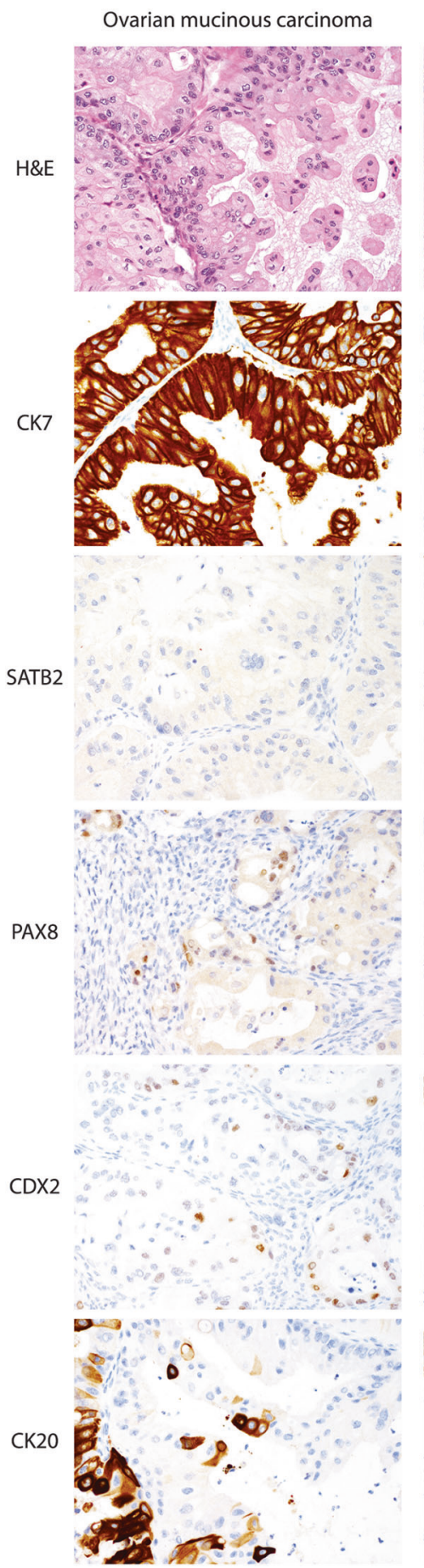

Fig. 1 Immunohistochemical stains First panel: typical staining pattern for an ovarian mucinous carcinoma: Hematoxylin and eosin (H\&E) stain, CK7 diffuse, SATB2 absent, PAX8 focal, CDX2 focal, CK20 focal. Second panel: typical staining pattern for low-grade appendiceal
Low-grade appendiceal neoplasm
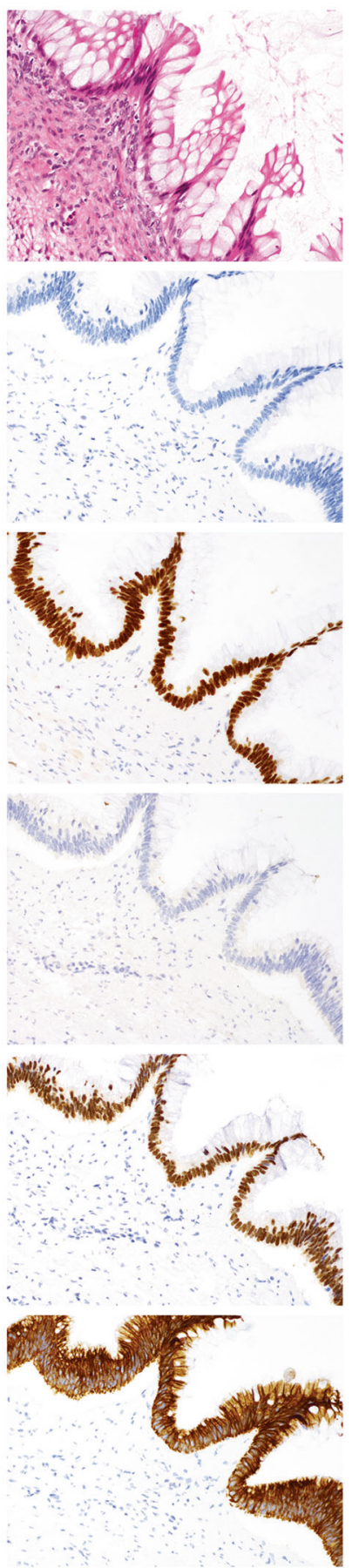

Colorectal carcinoma
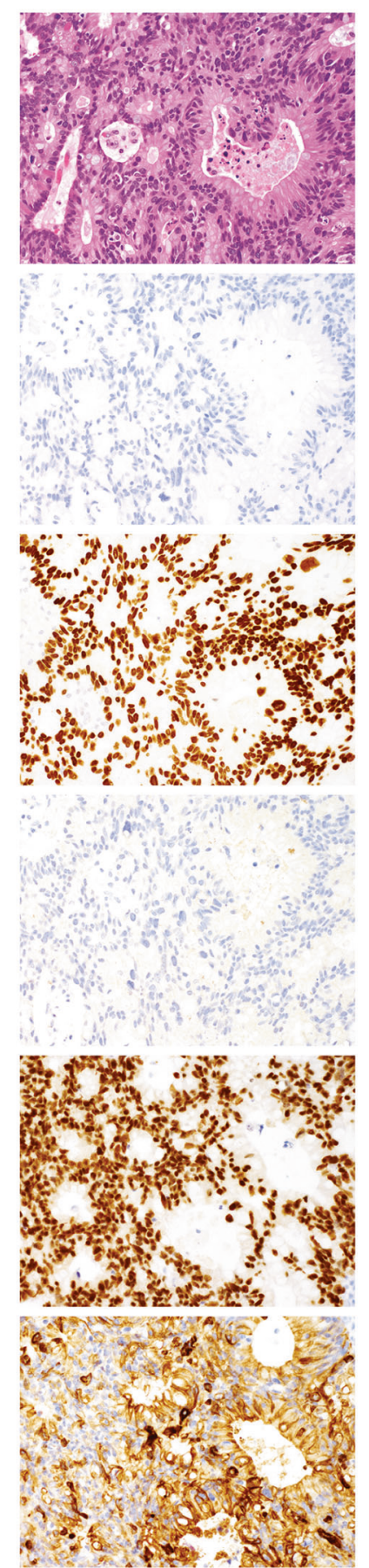

mucinous neoplasm: H\&E stain, CK7 absent, SATB2 diffuse, PAX8 absent, CDX2 diffuse, CK20 diffuse. Third panel: typical staining pattern for colorectal carcinoma: H\&E stain, CK7 absent, SATB2 diffuse, PAX8 absent, CDX2 diffuse, CK20 diffuse

potentially suggesting an incorrect original diagnosis of a primary ovarian tumor underwent a morphological review by a single gynecological pathologist (author MK). Rereview was performed on two representative full $\mathrm{H} \& \mathrm{E}$ 
sections to reassess the presence of the above-mentioned features of metastatic lower gastrointestinal adenocarcinoma.

\section{Statistical analyses}

The 3-tier scoring interpretation (absent/focal/diffuse) was categorized into 2 different binary datasets: the first cut-off was absent/present, with present including focal and diffuse staining, and the second cut-off was nondiffuse/diffuse with nondiffuse including absent and focal staining. Sensitivity, specificity, and balanced accuracy were calculated to assess test performance. Nominal logistic regression was used to rank the accuracy of different markers using the 3-tier scoring interpretation (absent/focal/diffuse) and binarized data.

All mucinous ovarian carcinomas from the OTTA consortium with available stage and survival data $(n=214)$ were used to investigate associations between SATB2 and PAX8 expression (absent/present) and overall survival. Survival was estimated using the Kaplan Meier method, and Cox proportional hazards regression adjusted for age, stage of disease, and cohort (test/expansion). Due to differences in study entry within the OTTA consortium, we applied lefttruncation to account for observation time at risk versus date of primary diagnosis. Survival analyses were censored at 10 years. All data management and sensitivity analyses were performed using SAS version 9.4. Survival analyses were conducted using R Studio and nominal logistic regression model in JMPv14 (SAS).

\section{Results}

\section{Performance of CK7, CK20, CDX2, SATB2 and PAX8 individually in the test cohort}

A test cohort consisted of 155 ovarian primary mucinous neoplasms and 230 neoplasms of lower gastrointestinal origin (Table 2, Supplementary Table S4). The ovarian primary mucinous neoplasms included 50 mucinous borderline tumors and 105 mucinous carcinomas. The gastrointestinal primaries were comprised of 123 Stage II colorectal adenocarcinomas and 107 appendiceal neoplasms including 39 goblet cell carcinomas, 24 low-grade appendiceal mucinous neoplasm, 20 carcinoids, 12 high grade appendiceal mucinous neoplasms and 12 non-mucinous adenocarcinomas.

The frequencies of 3-tier marker expression are shown in Table 2. Expression of CK20, CDX2, and SATB2 was present in almost all gastrointestinal primaries $(87 \%, 98 \%$ $90 \%$, respectively), while CK7 expression was detectable in almost all $(97 \%)$ and PAX8 in less than half $(45 \%)$ of ovarian mucinous neoplasms. Interestingly, $6(6 \%)$ of appendiceal cases (3 non-mucinous adenocarcinomas, 2 carcinoids and 1 goblet cell carcinoma) displayed diffuse
PAX8 expression (Supplementary Table S4), but this was not seen in any of the 123 colorectal adenocarcinomas (Table 2, Supplementary Table S4). Differences in CDX2 and CK20 expression were noted between ovarian mucinous borderline tumors and mucinous carcinomas, with carcinomas less likely to express CDX2. There was high concordance $(94 \%)$ between the two SATB2 antibodies tested (Supplementary Table S3).

Sensitivity, specificity and accuracy of individual markers in distinguishing ovarian mucinous neoplasms from gastrointestinal primaries are shown in Table 3. When the binary absence/presence cut-off is used, SATB2 shows the highest accuracy $(88.9 \%)$ among all the markers. However, when a binary nondiffuse/diffuse cut-off was used, CK7 achieved the highest accuracy (91.7\%). Notably, the nondiffuse/diffuse cut-off increased accuracy of CDX2 from 71 to $82 \%$, while the absence/presence cut-off showed the higher accuracy for PAX8 (76\% versus 63\%). CK20 showed the lowest accuracy only reaching $65 \%$ with the nondiffuse/ diffuse cut-off (Table 3).

\section{Performance of marker combinations in the test cohort}

We then tested the marker combinations with different inputs (3-tier, binary absence/presence cut-off, nondiffuse/ diffuse cut-off as well as different combinations of cut-offs) using nominal logistic regression modeling. Selected marker combinations arranged in descending order of accuracy are shown in Table 4 . The standard clinical panel consisting of CK7, CK20, and CDX2 with a binary absent/present cut off shows the same accuracy as CK7 alone (Tables 3 and 4: $87.5 \%)$. Once SATB2 and PAX8 are added to make a 5marker panel, the accuracy increases to $95.3 \%$. The effect was more pronounced for the distinction of appendiceal from ovarian (93.1\% versus $86.6 \%$ ) compared to colorectal primaries $(94.6 \%$ versus $93.9 \%)$. Removal of CK20 or CK20 and CDX2 only slightly affected AUC values for distinguishing gastrointestinal from ovarian tumors $(95.1 \%$, $94.3 \%$, respectively).

Despite the fact that the binary nondiffuse/diffuse cut-off generally showed a higher accuracy compared to the absent/ present cut-off for individual markers, this did not translate into higher accuracy for marker combinations. For example, the best two individual marker cut-offs (CK7 nondiffuse, SATB2 present) performed slightly worse than CK7 absent, SATB2 present. This is because SATB2 does not add information to CK7 nondiffuse alone. However, when the interpretation was left to 3-tier (absent, focal, diffuse), the two-marker combination of CK7 and SATB2 performed as well as a five-marker combination. A decision tree for the 3tier interpretation of the CK7/SATB2 combination is shown in Fig. 2. 
Table 2 Frequency of marker expression in ovarian and lower gastrointestinal tumors

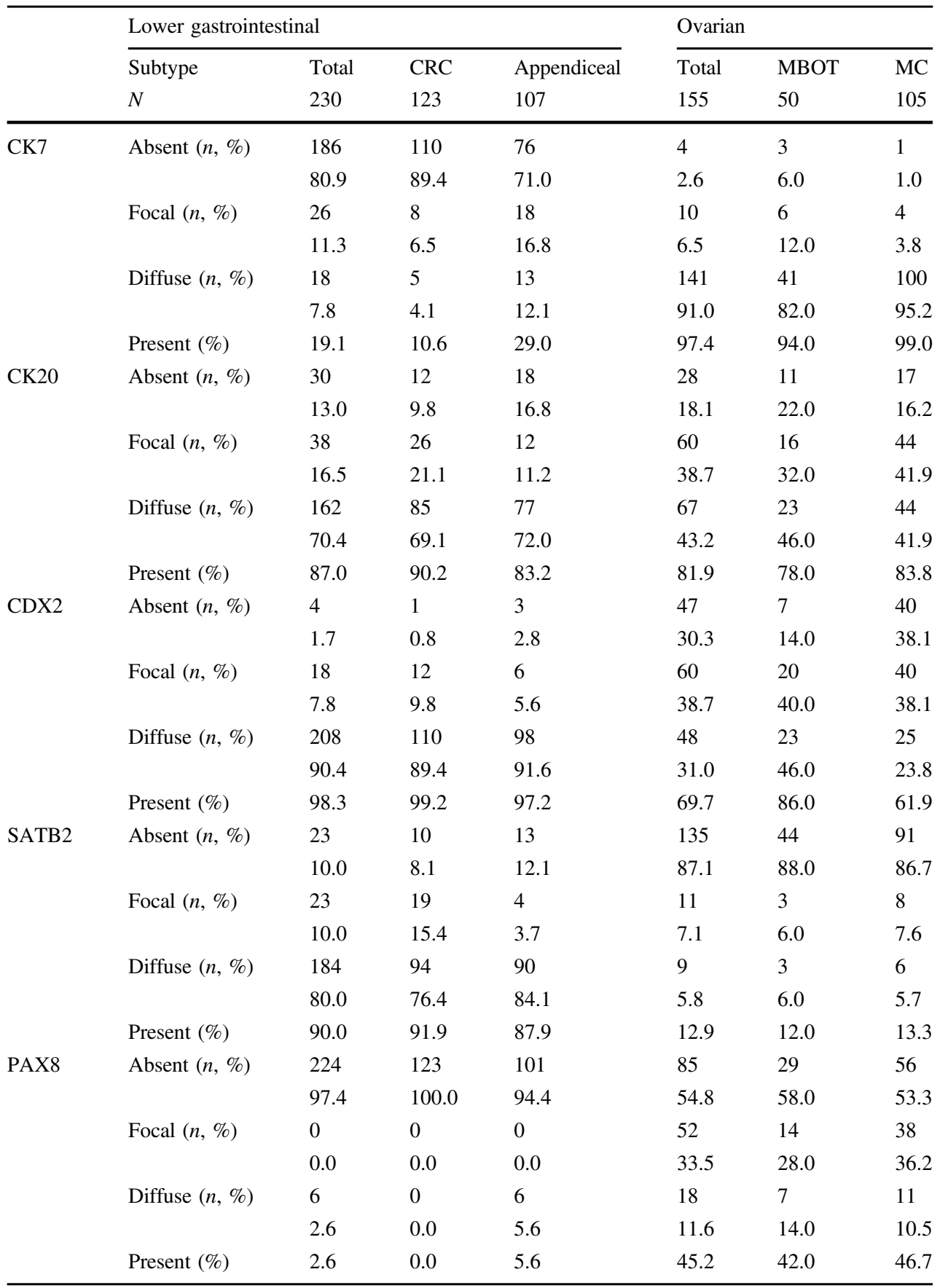

\section{Re-evaluation of ovarian outlier cases using CK7/ SATB2 combination in the test cohort}

We performed a focused clinical and morphological reevaluation on the 11 primary ovarian mucinous carcinomas with aberrant CK7/SATB2 staining from Fig. 2 (4 CK7 absent and 7 SATB2 diffusely positive ovarian mucinous tumors, supplementary table 5). During follow up, two of the eleven patients survived between 5 and 10 years, and 4 patients survived $>10$ years. The long survival time of these 6 patients is consistent with the classification of ovarian primaries. Four patients died of their disease within 2 years. Among these, two PAX8 negative cases had associated teratomas supporting primary ovarian origin. The other two cases were both PAX8 positive, also supporting an ovarian primary; one case showed anaplastic carcinoma within a mural nodule in a background of a mucinous borderline tumor and the other showed multifocal destructive invasive mucinous carcinoma. The last patient was lost to follow-up after 
Table 3 Sensitivity, specificity and balanced accuracy of individual markers to predict a lower GI tumor in the test and expansion cohorts using different cut-offs

\begin{tabular}{lcclllllllll}
\hline Test cohort & & & & & & & & & & & \\
\hline $\begin{array}{l}\text { Binary criteria (absent versus } \\
\text { present) }\end{array}$ & Sensitivity & $95 \%$ & CI & Specificity & $95 \%$ & CI & Accuracy & $95 \%$ CI \\
SATB2 present & 0.90 & 0.85 & 0.94 & 0.87 & 0.81 & 0.92 & 0.89 & 0.85 & 0.92 \\
CK7 absent & 0.81 & 0.75 & 0.86 & 0.97 & 0.94 & 0.99 & 0.88 & 0.84 & 0.91 \\
PAX8 absent & 0.97 & 0.94 & 0.99 & 0.45 & 0.37 & 0.53 & 0.76 & 0.72 & 0.81 \\
CDX2 present & 0.98 & 0.96 & 1.00 & 0.30 & 0.23 & 0.38 & 0.71 & 0.66 & 0.75 \\
CK20 present & 0.87 & 0.82 & 0.91 & 0.18 & 0.12 & 0.25 & 0.59 & 0.54 & 0.64 \\
Binary criteria (diffuse versus & non-diffuse) & & & & & & & & \\
CK7 nondiffuse & 0.92 & 0.88 & 0.95 & 0.91 & 0.85 & 0.95 & 0.92 & 0.88 & 0.94 \\
SATB2 diffuse & 0.80 & 0.74 & 0.85 & 0.94 & 0.86 & 0.97 & 0.86 & 0.82 & 0.89 \\
CDX2 diffuse & 0.81 & 0.76 & 0.86 & 0.83 & 0.75 & 0.89 & 0.82 & 0.78 & 0.86 \\
PAX8 nondiffuse & 0.97 & 0.94 & 0.99 & 0.12 & 0.07 & 0.18 & 0.63 & 0.58 & 0.68 \\
CK20 diffuse & 0.71 & 0.64 & 0.77 & 0.56 & 0.48 & 0.64 & 0.65 & 0.60 & 0.70 \\
Expansion cohort & & & & & & & & & \\
Binary criteria (absent versus present) & & & & & & & & & & \\
SATB2 present & 0.95 & 0.91 & 0.97 & 0.88 & 0.83 & 0.92 & 0.92 & 0.88 & 0.94 \\
PAX8 absent & 0.97 & 0.94 & 0.99 & 0.41 & 0.35 & 0.49 & 0.71 & 0.67 & 0.75 \\
Binary criteria (diffuse versus non-diffuse) & & & & & & & & \\
SATB2 diffuse & 0.80 & 0.74 & 0.85 & 0.94 & 0.89 & 0.97 & 0.96 & 0.83 & 0.90 \\
PAX8 nondiffuse & 0.97 & 0.94 & 0.99 & 0.14 & 0.09 & 0.19 & 0.58 & 0.53 & 0.63 \\
\hline
\end{tabular}

Table 4 Accuracy of markers in combination to predict a lower gastrointestinal primary

\begin{tabular}{|c|c|c|c|c|c|c|c|c|}
\hline \multirow[b]{2}{*}{ Rank } & \multirow[b]{2}{*}{ Markers (N) } & \multirow[b]{2}{*}{ Marker combination assessed } & \multicolumn{2}{|c|}{ GI vs Ov } & \multicolumn{2}{|c|}{$\mathrm{CRC}$ vs Ov } & \multicolumn{2}{|c|}{ App vs Ov } \\
\hline & & & ROC & Accuracy & ROC & Accuracy & ROC & Accuracy \\
\hline 1 & 5 & CK7 3-tier, SATB2 3-tier, CDX2 3-tier, PAX8 3-tier, CK20 3-tier & 0.981 & 95.3 & 0.988 & 96.0 & 0.976 & 94.7 \\
\hline 2 & 4 & CK7 3-tier, SATB2 3-tier, CDX2 3-tier, PAX8 3-tier & 0.978 & 95.8 & 0.986 & 95.7 & 0.973 & 94.7 \\
\hline 3 & 3 & CK7 3-tier, SATB2 3-tier, PAX8 3-tier & 0.978 & 95.6 & 0.986 & 95.7 & 0.969 & 93.9 \\
\hline 4 & 3 & CK7 3-tier, SATB2 3-tier, CDX2 3-tier & 0.976 & 95.6 & 0.986 & 95.7 & 0.966 & 93.5 \\
\hline 5 & 2 & CK7 3-tier, SATB2 3-tier & 0.973 & 95.3 & 0.984 & 95.3 & 0.957 & 93.5 \\
\hline 6 & 5 & $\begin{array}{l}\text { SATB2 present, CK7 absent, PAX8 absent, CDX2 present, CK20 } \\
\text { present }\end{array}$ & 0.972 & 95.3 & 0.983 & 94.6 & 0.96 & 93.1 \\
\hline 7 & 4 & SATB 2 present, CK7 absent, PAX8 absent, CDX2 present & 0.972 & 95.1 & 0.982 & 96.0 & 0.959 & 92.0 \\
\hline 8 & 3 & SATB2 present, CK7 absent, PAX8 absent & 0.97 & 94.3 & 0.981 & 95.0 & 0.957 & 91.6 \\
\hline 9 & 3 & CK7 3-tier, CDX2 3-tier, CK20 3-tier & 0.97 & 93.2 & 0.98 & 95.0 & 0.962 & 91.6 \\
\hline 10 & 2 & SATB2 present, CK7 absent & 0.963 & 93.5 & 0.979 & 93.9 & 0.945 & 90.5 \\
\hline 11 & 2 & CK7 3-tier, CK20 3-tier & 0.956 & 92.2 & 0.968 & 93.9 & 0.939 & 90.5 \\
\hline 12 & 2 & CK7 nondiffuse, SATB2 present & 0.953 & 91.7 & 0.963 & 93.2 & 0.942 & 89.7 \\
\hline 13 & 3 & CK7 absent, CDX2 present, CK20 present (clinical standard) & 0.922 & 87.5 & 0.954 & 93.9 & 0.886 & 86.6 \\
\hline
\end{tabular}

GI gastrointestinal, $O v$ ovarian, $C R C$ colorectal, App appendiceal, ROC Receiver Operating Characteristic

22 months but was alive at last contact. This tumor was CK7 focal, SATB2 diffuse, PAX8 absent, CDX2 diffuse, and CK20 diffuse. This was the only suspected misclassified case in the test cohort, which was originally diagnosed as an ovarian primary but re-evaluation suggested a lower gastrointestinal metastasis.

\section{Frequency of SATB2 and PAX8 expression across five main ovarian carcinoma histotypes in the expansion cohort}

Given the high performance of SATB2 (90\% sensitivity, $87 \%$ specificity) in distinguishing primary ovarian mucinous 


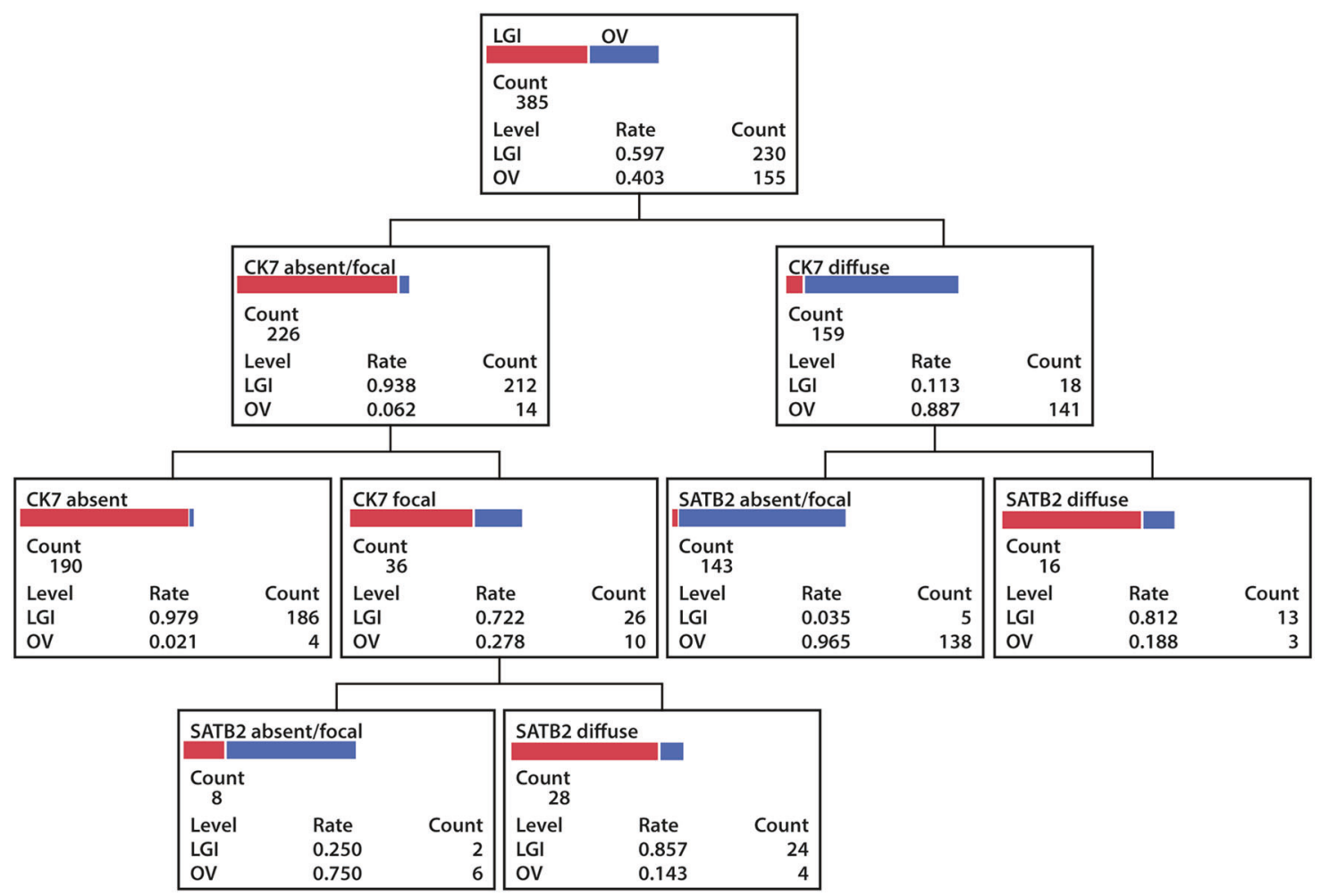

Fig. 2 Decision tree for the 3-tier interpretation of the CK7/SATB2 combination: If CK7 is absent, a lower gastrointestinal (LGI) primary (red bar) is most likely regardless of the staining pattern of SATB2. If CK7 is focal and SATB2 is diffuse, a lower gastrointestinal primary should be favored. The combination of focal CK7 and absent/focal

neoplasms from lower gastrointestinal primaries as an individual marker, we also investigated the frequency of SATB2 expression in an expansion set of tumors from the remaining OTTA cohort. This contained additional ovarian mucinous neoplasms $(n=205, n=159$ invasive and $n=46$ borderline), as well as 2671 ovarian carcinomas of other histotypes. SATB2 expression frequencies for mucinous carcinomas were similar between the test and expansion sets (Table 5), however SATB2 was more frequently present in the borderline tumors of the test set, although not statistically significant ( $12 \%$ versus $4 \%, p=0.3$ ).

In the other ovarian carcinoma histotypes, we observed the highest frequency of positivity in endometrioid carcinomas with $13 \%$ showing focal and $2 \%$ diffuse SATB2 staining. In total, $4 \%$ of high-grade serous and clear cell carcinomas also expressed SATB2. All these ovarian carcinoma histotypes showed a high frequency of PAX8 expression. Only very rare cases of endometrioid (3\%), and high grade serous $(1 \%)$ carcinoma showed an aberrant expression pattern of SATB2 + /PAX8- (Table 5). Thus, the inclusion of PAX8 should aid in the distinction of these ovarian tumors, especially for SATB2 + endometrioid ovarian carcinoma from gastrointestinal metastases.
SATB2 suffers from low numbers and second line markers should be considered. If CK7 is diffuse and SATB2 is absent or focal, this represents an ovarian $(\mathrm{OV})$ primary (blue bar) with $96 \%$ probability. However, if both CK7 and SATB2 are diffuse, this scenario more likely represents a lower gastrointestinal primary

Table 3 also shows the validation of SATB2 and PAX8 in mucinous tumors from the expansion cohort, with an accuracy of $92 \%$ at an absent/present cut-off, and 95\% when using a nondiffuse/diffuse cut-off. The low specificity of PAX8 absent to predict a gastrointestinal tumor produced accuracy of only $71 \%$.

\section{Prognostic significance of SATB2 and PAX8 in all ovarian mucinous carcinomas from the OTTA consortium}

As expected, the 5-year overall survival significantly differs between low (I/II) $80 \%$, versus high stage mucinous carcinomas (III/IV) $17 \%(p<0.0001)$ (Fig. 3a). Since a subset of ovarian mucinous carcinomas (13\%) expressed SATB2 (Tables 2 and 3), we explored an association with survival and performed a Cox regression adjusted for patient age, disease stage, and cohort, and the proportional hazards assumption was not violated. We observed a significant association for SATB2 expression and poorer overall survival (Hazard ratio 2.49 (95\% CI 1.22-5.09), $p=0.01$ ) (Fig. 3b). Expression of PAX8 was not associated with survival $(p=0.3$, HR $0.76(0.44-1.32))$ (Fig. 3c). 


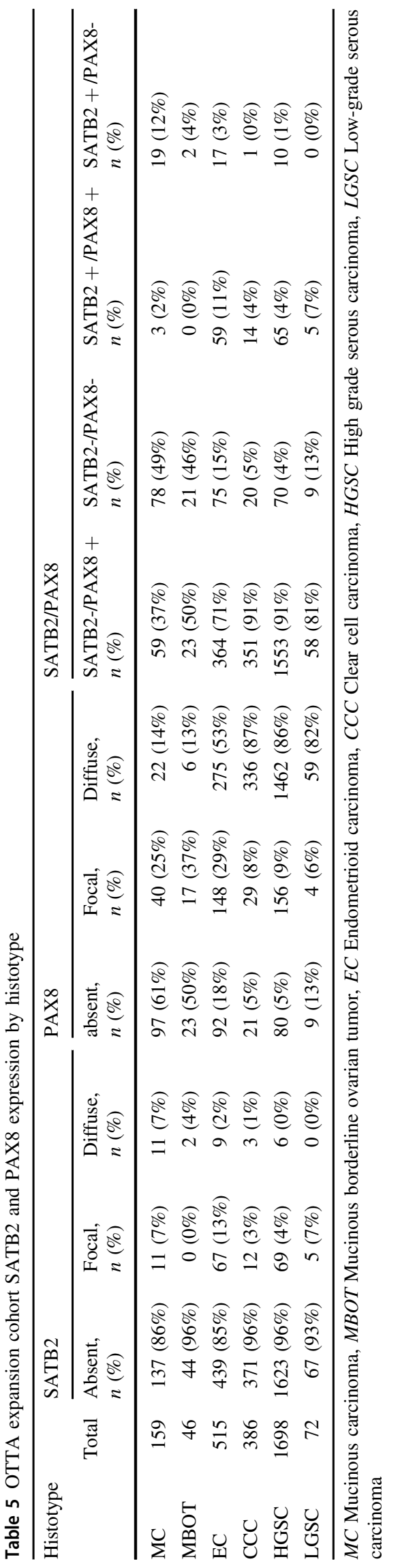

\section{Discussion}

Herein, we show that a combination of CK7 and SATB2 using a 3-tier interpretation (absent/focal/diffuse) is the most efficient ancillary test to distinguish primary ovarian mucinous neoplasms from metastatic lower gastrointestinal primary tumors. This represents a refinement to previous recommendations for the use of different permutations of the five markers (CK7, CK20, CDX2, SATB2, and PAX8) in routine clinical practice [5-7]. We also validated the specificity of SATB2 in the largest series of ovarian tumor tissue available to-date internationally.

It has long been known that CK7 is the best single discriminatory marker for lower gastrointestinal primaries compared to ovarian mucinous neoplasms [20]. While CK7 is diffusely expressed in almost all ovarian primaries, it is largely absent in colorectal adenocarcinoma but can be expressed in $B R A F$-mutated mismatch repair proficient colorectal adenocarcinomas [21]. Its specificity towards appendiceal neoplasms is, however, limited. The combination of CK7 with CDX2 has been promoted particularly in a 3-tier staining distribution (absent/focal/diffuse) [12]. In line with these suggestions, we show that increasing the cut-off for interpretation increases accuracy of CDX2 by $>10 \%$. Despite several publications questioning the specificity of CK20 [3], its use in routine clinical practice remains high. Based on our findings and those of previous publications $[3,4,12]$, we do not recommend the use of CK20 to distinguish lower gastrointestinal from ovarian mucinous neoplasms. We propose that CK20 could be replaced by SATB2 to increase accuracy in a cost neutral way.

In publicly funded health care systems, finding the most efficient marker combinations to enable accurate tumor diagnosis is essential to deliver value-based care. Using a larger number of cases, we validated previous studies showing good performance for SATB2 [6]. These results warrant adding SATB2 to the immunohistochemical arsenal. Other studies suggested that SATB2 is not optimally sensitive or specific when used as single marker $[6,9]$. SATB2 has also been shown to have superior value in distinguishing certain lower from upper gastrointestinal metastasis [22, 23]. Of note, upper gastrointestinal metastasis can have the same immunohistochemical profile as ovarian mucinous carcinomas including some pancreatic adenocarcinoma showing PAX8 expression [24]. Herein, we found a higher sensitivity of SATB2 alone for colorectal adenocarcinomas $(91.6 \%)$ and for low-grade appendiceal mucinous neoplasms $(97.8 \%)$ compared to those reported by Moh et al. (71.3\% and 80\%, respectively) [6]. The higher SATB2 expression frequency for ovarian mucinous neoplasms both in our testing and expansion cohorts (12\%) compared to previously reported (5\%) raises the possibility of an influx of misclassified lower gastrointestinal 

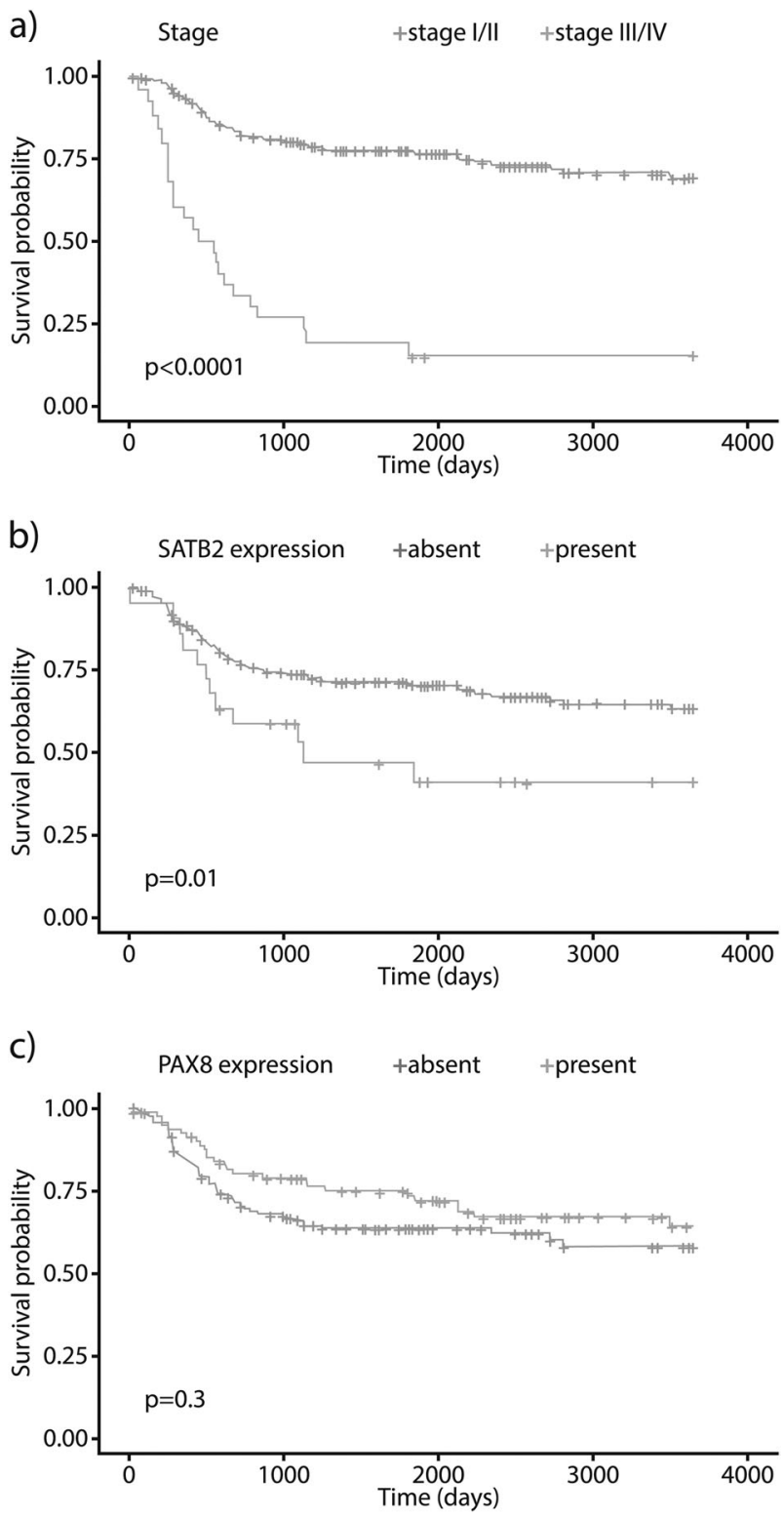

Fig. 3 a-c Kaplan-Meier overall survival curves. a Overall survival in women diagnosed with mucinous carcinomas $(n=214)$ by Stage $(\mathrm{I} / \mathrm{II}$ versus III/IV). b Overall survival in women diagnosed with mucinous carcinomas $(n=214)$ by SATB2 expression (absent/present). c Overall survival in women diagnosed with mucinous carcinomas $(n=$ 214) by PAX8 expression (absent/present)

primaries. Re-evaluation of outlier cases from the testing cohort revealed that only a single case was a likely misclassified metastatic gastrointestinal primary while others were teratoma-associated ovarian mucinous neoplasms or anaplastic carcinomas presenting as mural nodules in mucinous tumors. Ovarian mucinous tumors associated with teratomas, which account for $\sim 5 \%$ of ovarian mucinous tumors [25], have the same immunoprofile as those that originate in the gastrointestinal tract, including SATB2 expression. The distinction in this scenario would rely on the identification of the teratoma. Although we could not reevaluate outlier cases in the multi-institutional expansion cohort, we believe, that given the similar frequency of SATB2 expression in ovarian mucinous neoplasms in our extensively reviewed test and expansion cohorts, this provides a more realistic estimate of the SATB2 expression frequency in ovarian mucinous tumors (12\%). Furthermore, the 5-year survival estimates of mucinous carcinomas in OTTA $(80 \%$ for Stage I/II and $17 \%$ for Stage III/IV, Fig. 3a) were similar to the SEER database $(83 \%$ for localized, $69.5 \%$ for regional spread and $14 \%$ for distant metastases) [26]. Overall, this argues against a major component of misclassified metastatic lower gastrointestinal tract neoplasms within the OTTA cohort and the relatively high proportion of primary mucinous carcinomas could be explained by study sites selectively enriching for rare tumor histotypes.

While CK7 and SATB2 together make the most efficient panel, CDX2 and PAX8 are reasonable second line markers. In particular, the specificity of PAX8 helps to rule out a lower gastrointestinal primary, despite the potential pitfalls: we found that some appendiceal neoplasms exhibit diffuse PAX8 expression, and the frequency of PAX8 expression in ovarian mucinous tumors is much lower and often only focal when compared to the other ovarian carcinoma histotypes [5].

The differential diagnosis between an ovarian primary and a lower GI metastasis is frequently ovarian endometrioid carcinoma. SATB2 was expressed in $15 \%$ of endometrioid carcinoma with the majority showing focal expression. We noted that SATB2 expression was commonly seen in squamous morules but did not quantify this observation. This quantitation was done in a recent study reporting a correlation of SATB2 expression and squamous morules but noting that SATB2 expression occasionally occurred outside of squamous morules [27]. This staining pattern is similar to CDX2, which is expressed at an even higher frequency $(\sim 50 \%)$ in ovarian endometrioid carcinomas, also commonly in squamous morules [28]. Ovarian endometrioid carcinomas express PAX8 and ER at a higher percentage ( $\sim 85 \%)$ compared to mucinous carcinomas [29]. Therefore, the panel to distinguish ovarian endometrioid carcinomas from lower gastrointestinal metastasis should be wider including CK7, ER, PAX8 and SATB2 with SATB2 being more specific than CDX2. Of note, SATB2 is not entirely specific with regard to high-grade serous and clear cell carcinomas because almost $5 \%$ of these tumors did show at least focal expression. In this context, it is also noteworthy that the osteosarcoma component of carcinosarcomas can express SATB2 [30].

Our observation of an adverse survival association with SATB2 expression within mucinous carcinomas is intriguing. Despite our relatively large sample size, it is possible 
that this result could be still a false positive. It does raise the possibility that SATB2 expressing ovarian mucinous carcinomas such as teratoma-associated or anaplastic carcinomas might be associated with a slightly worse outcome, although the current literature is very limited in this area [25]. The finding contrasts with previous reports of a survival benefit with SATB2 expression in colorectal cancer [31]. We did not observe differences in overall survival based on the PAX8 expression status arguing against a biological split of PAX8 positive versus negative mucinous carcinoma.

In the future, molecular studies may assist in further refining classification. However, currently mutational profiles do not seem to achieve sensitivities and specificities of the biomarkers assessed here. For example, TP53 or KRAS mutations are present in both ovarian mucinous and colorectal carcinomas and even though $A P C$ mutations are absent in ovarian mucinous carcinomas, and high in colorectal adenocarcinomas, this is limited to non-mucinous adenocarcinomas ( $88 \%$ vs $24 \%$ mucinous) [32]. In addition, small numbers of mutations in GNAS have been reported in mucinous ovarian tumors, as well as in a subset of lowgrade appendiceal mucinous neoplasms [33-36].

\section{Conclusion}

The immunohistochemical profile of most "intestinal-type" primary ovarian mucinous primaries is distinct from lower gastrointestinal neoplasms. Our study provides strong evidence that SATB2 is a better marker than CK20 for the distinction of ovarian mucinous neoplasms from colorectal carcinomas and we recommend replacement of CK20 by SATB2. In combination, CK7 and SATB2 efficiently distinguish ovarian mucinous primaries from lower gastrointestinal metastasis, particularly if the distribution of staining in a 3-tier system is considered.

Acknowledgements This study is supported by research funds from Cancer Research Society of Canada (19319). NSM is supported by the NSW Ministry of Health and UNSW Sydney under the NSW Health PhD Scholarship Program, and the Translational Cancer Research Network, a translational cancer research center program funded by the Cancer Institute NSW. The Gynaecological Oncology Biobank at Westmead was funded by Cancer Institute NSW (12/RIG/1-17 and 15/ RIG/1-16) and the National Health and Medical Research Council of Australia (ID310670, ID628903). FM is funded by University of Pittsburgh School of Medicine Dean's Faculty Advancement Award. The HOPE study is funded by: US National Cancer Institute (K07CA80668, P50-CA159981, R01CA095023), US Army Medical Research and Materiel Command (DAMD17-02-1-0669) and NIH/ National Center for Research Resources/General Clinical Research Center (MO1- RR000056). KS is funded by the Swedish Cancer foundation. The Generations Study thank Breast Cancer Now, the Institute of Cancer Research and Ovarian Cancer Action for support and funding. The ICR acknowledge NHS funding to the NIHR
Biomedical Research Centre. Tissue samples for GER were provided by the tissue bank of the National Center for Tumor Diseases (NCT, Heidelberg, Germany) in accordance with the regulations of the tissue bank and the approval of the ethics committee of the University of Heidelberg. The Health Science Alliance (HSA) Biobank acknowledges the UNSW Biorepository, UNSW Sydney, Australia. We thank Shuhong Liu, Young Ou, and Deon Richards for immunohistochemical stains, and Thomas Kryton, BFA, digital imaging specialist for Alberta Public Lab for creating the figures. We especially thank all the study participants, health care staff and data providers internationally who have made this research possible.

\section{Compliance with ethical standards}

Conflict of interest The authors declare that they have no conflict of interest.

Publisher's note: Springer Nature remains neutral with regard to jurisdictional claims in published maps and institutional affiliations.

\section{References}

1. Lee KR, Young RH. The distinction between primary and metastatic mucinous carcinomas of the ovary: gross and histologic findings in 50 cases. Am J Surg Pathol. 2003;27:281-92.

2. Stewart CJ, Ardakani NM, Doherty DA, Young RH. An evaluation of the morphologic features of low-grade mucinous neoplasms of the appendix metastatic in the ovary, and comparison with primary ovarian mucinous tumors. Int J Gynecol Pathol. 2014;33:1-10.

3. Vang R, Gown AM, Wu LS, Barry TS, Wheeler DT, Yemelyanova $\mathrm{A}$, et al. Immunohistochemical expression of CDX2 in primary ovarian mucinous tumors and metastatic mucinous carcinomas involving the ovary: comparison with CK20 and correlation with coordinate expression of CK7. Mod Pathol. 2006;19:1421-8.

4. McCluggage WG. Immunohistochemistry in the distinction between primary and metastatic ovarian mucinous neoplasms. J Clin Pathol. 2012;65:596-600.

5. Ates Ozdemir D, Usubutun A. PAX2, PAX8 and CDX2 expression in metastatic mucinous, primary ovarian mucinous and seromucinous tumors and review of the literature. Pathol Oncol Res. 2016;22:593-9.

6. Moh M, Krings G, Ates D, Aysal A, Kim GE, Rabban JT. SATB2 expression distinguishes ovarian metastases of colorectal and appendiceal origin from primary ovarian tumors of mucinous or endometrioid type. Am J Surg Pathol. 2016;40:419-32.

7. Strickland S, Parra-Herran C. Immunohistochemical characterization of appendiceal mucinous neoplasms and the value of special AT-rich sequence-binding protein 2 in their distinction from primary ovarian mucinous tumours. Histopathology. 2016;68:977-87.

8. Dobreva G, Chahrour M, Dautzenberg M, Chirivella L, Kanzler B, Farinas I, et al. SATB2 is a multifunctional determinant of craniofacial patterning and osteoblast differentiation. Cell. 2006;125:971-86.

9. Magnusson K, de Wit M, Brennan DJ, Johnson LB, McGee SF, Lundberg E, et al. SATB2 in combination with cytokeratin 20 identifies over $95 \%$ of all colorectal carcinomas. Am J Surg Pathol. 2011;35:937-48.

10. Ozcan A, Shen SS, Hamilton C, Anjana K, Coffey D, Krishnan B, et al. PAX 8 expression in non-neoplastic tissues, primary tumors, and metastatic tumors: a comprehensive immunohistochemical study. Mod Pathol. 2011;24:751-64. 
11. Tabrizi AD, Kalloger SE, Kobel M, Cipollone J, Roskelley CD, Mehl E. Primary ovarian mucinous carcinoma of intestinal type: significance of pattern of invasion and immunohistochemical expression profile in a series of 31 cases. Int J Gynecol Pathol. 2010;29:99-107.

12. Groisman GM, Meir A, Sabo E. The value of Cdx2 immunostaining in differentiating primary ovarian carcinomas from colonic carcinomas metastatic to the ovaries. Int $\mathrm{J}$ Gynecol Pathol. 2004;23:52-7.

13. Zaino RJ, Brady MF, Lele SM, Michael H, Greer B, Bookman MA. Advanced stage mucinous adenocarcinoma of the ovary is both rare and highly lethal: a Gynecologic Oncology Group study. Cancer. 2011;117:554-62.

14. Rambau PF, Vierkant RA, Intermaggio MP, Kelemen LE, Goodman MT, Herpel E, et al. Association of p16 expression with prognosis varies across ovarian carcinoma histotypes: an Ovarian Tumor Tissue Analysis consortium study. J Pathol Clin Res. 2018;4:250-61.

15. Ovarian Tumor Tissue Analysis C, Goode EL, Block MS, Kalli KR, Vierkant RA, Chen W, et al. Dose-Response Association of CD8+Tumor-Infiltrating Lymphocytes and Survival Time in High-Grade Serous Ovarian Cancer. JAMA Oncol. 2017;3: e173290.

16. Kobel M, Madore J, Ramus SJ, Clarke BA, Pharoah PD, Deen S, et al. Evidence for a time-dependent association between FOLR1 expression and survival from ovarian carcinoma: implications for clinical testing. An Ovarian Tumour Tissue Analysis consortium study. Br J Cancer. 2014;111:2297-307.

17. Sieh W, Kobel M, Longacre TA, Bowtell DD, deFazio A, Goodman MT, et al. Hormone-receptor expression and ovarian cancer survival: an Ovarian Tumor Tissue Analysis consortium study. Lancet Oncol. 2013;14:853-62.

18. Gui X, Meng Z, McConnell YJ, Liu S, Falck VG, Mack LA, et al. Differing expression profiles of Notch/enterocyte and Wnt/secretory lineage signallings are associated with morphological diversity of appendiceal tumours. J Clin Pathol. 2017;70:40-50.

19. Kobel M, Rahimi K, Rambau PF, Naugler C, Le Page C, Meunier $\mathrm{L}$, et al. An immunohistochemical algorithm for ovarian carcinoma typing. Int J Gynecol Pathol. 2016;35:430-41.

20. Kelemen LE, Kobel M. Mucinous carcinomas of the ovary and colorectum: different organ, same dilemma. Lancet Oncol. 2011;12:1071-80.

21. Landau MS, Kuan SF, Chiosea S, Pai RK. BRAFmutated microsatellite stable colorectal carcinoma: an aggressive adenocarcinoma with reduced CDX2 and increased cytokeratin 7 immunohistochemical expression. Hum Pathol. 2014;45: 1704-12.

22. Yang C, Sun L, Zhang L, Zhou L, Zhao M, Peng Y, et al. Diagnostic utility of SATB2 in metastatic Krukenberg tumors of the ovary: an immunohistochemical study of 70 cases with comparison to CDX2, CK7, CK20, chromogranin, and synaptophysin. Am J Surg Pathol. 2018;42:160-71.
23. Ma C, Lowenthal BM, Pai RK. SATB2 is superior to CDX2 in distinguishing signet ring cell carcinoma of the upper gastrointestinal tract and lower gastrointestinal tract. Am J Surg Pathol. 2018;42:1715-22.

24. Tacha D, Zhou D, Cheng L. Expression of PAX8 in normal and neoplastic tissues: a comprehensive immunohistochemical study. Appl Immunohistochem Mol Morphol. 2011;19:293-9.

25. Black JD, Roque DM, Pasternak MC, Buza N, Rutherford TJ, Schwartz PE, et al. A series of malignant ovarian cancers arising from within a mature cystic teratoma: a single institution experience. Int J Gyneacol Cancer. 2015;25:792-7.

26. Peres LC, Cushing-Haugen KL, Kobel M, Harris HR, Berchuck A, Rossing MA, et al. Invasive epithelial ovarian cancer survival by histotype and disease stage. J Natl Cancer Inst. 2018;111:60-8.

27. Le Page C, Kobel M, Meunier L, Provencher DM, Mes-Masson AM, Rahimi K. A COEUR cohort study of SATB2 expression and its prognostic value in ovarian endometrioid carcinoma. $\mathrm{J}$ Pathol Clin Res. 2019. https://doi.org/10.1002/cjp2.131.

28. Wang L, Rambau PF, Kelemen LE, Anglesio MS, Leung S, Talhouk A, et al. Nuclear beta-catenin and CDX2 expression in ovarian endometrioid carcinoma identify patients with favourable outcome. Histopathology. 2019;74:452-62.

29. Rambau P, Kelemen LE, Steed H, Quan ML, Ghatage P, Kobel M. Association of hormone receptor expression with survival in ovarian endometrioid carcinoma: biological validation and clinical implications. Int J Mol Sci. 2017;18:515.

30. Sangoi AR, Kshirsagar M, Horvai AE, Roma AA. SATB2 expression is sensitive but not specific for osteosarcomatous components of gynecologic tract carcinosarcomas: a clinicopathologic study of 60 cases. Int J Gynecol Pathol. 2017;36:140-5.

31. Eberhard J, Gaber A, Wangefjord S, Nodin B, Uhlén M, Ericson Lindquist K, et al. A cohort study of the prognostic and treatment predictive value of SATB2 expression in colorectal cancer. Br J Cancer. 2012;106:931.

32. Song GA, Deng G, Bell I, Kakar S, Sleisenger MH, Kim YS. Mucinous carcinomas of the colorectum have distinct molecular genetic characteristics. Int J Oncol. 2005;26:745-50.

33. Crobach S, Ruano D, van Eijk R, Schrumpf M, Fleuren G, van Wezel $\mathrm{T}$, et al. Somatic mutation profiles in primary colorectal cancers and matching ovarian metastases: Identification of driver and passenger mutations. J Pathol Clin Res. 2016;2:166-74.

34. Nishikawa G, Sekine S, Ogawa R, Matsubara A, Mori T, Taniguchi $\mathrm{H}$, et al. Frequent GNAS mutations in low-grade appendiceal mucinous neoplasms. Br J Cancer. 2013;108:951-8.

35. Meagher NS, Schuster K, Voss A, Budden T, Pang CNI, deFazio A, et al. Does the primary site really matter? Profiling mucinous ovarian cancers of uncertain primary origin (MO-CUP) to personalise treatment and inform the design of clinical trials. Gynecol Oncol. 2018;150:527-33.

36. Ryland GL, Hunter SM, Doyle MA, Caramia F, Li J, Rowley SM, et al. Mutational landscape of mucinous ovarian carcinoma and its neoplastic precursors. Genome Med. 2015;7:87.

\section{Affiliations}

Nicola S. Meagher $\mathbb{D}^{1,2,3} \cdot$ Linyuan Wang ${ }^{4}$ Peter F. Rambau ${ }^{4,5} \cdot$ Maria P. Intermaggio $^{1,3} \cdot$ David G. Huntsman $^{6,7,8}$. Lynne R. Wilkens ${ }^{9} \cdot$ Mona A. El-Bahrawy ${ }^{10} \cdot$ Roberta B. Ness $^{11} \cdot$ Kunle Odunsi $^{12}$. Helen Steed ${ }^{13} \cdot$ Esther Herpel $^{14,15}$. Michael S. Anglesio ${ }^{8}$ - Bonnie Zhang ${ }^{16} \cdot$ Neil Lambie $^{17}$. Anthony J. Swerdlow ${ }^{18,19}$ - Jan Lubiński ${ }^{20}$. Robert A. Vierkant ${ }^{21}$ - Ellen L. Goode ${ }^{22} \cdot$ Usha Menon $^{23} \cdot$ Aleksandra Toloczko-Grabarek $^{20}$ - Oleg Oszurek ${ }^{24}$.

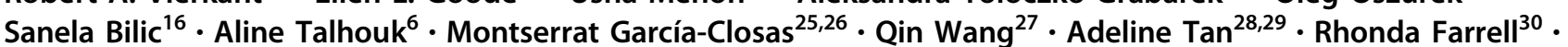
Catherine J. Kennedy ${ }^{31,32} \cdot$ Mercedes Jimenez-Linan $^{33} \cdot$ Karin Sundfeldt $^{34,35} \cdot$ John L. Etter ${ }^{36} \cdot$ Janusz Menkiszak $^{37}$. Marc T. Goodman ${ }^{38,39}$ • Paul Klonowski ${ }^{4}$ Yee Leung ${ }^{40}$. Stacey J. Winham ${ }^{21} \cdot$ Kirsten B. Moysich $^{36}$. 
Sabine Behrens ${ }^{41} \cdot$ Tomasz Kluz $^{42} \cdot$ Robert P. Edwards $^{43,44}$ - Jacek Gronwald ${ }^{20}$ - Francesmary Modugno ${ }^{44,45}$. Brenda Y Hernandez ${ }^{46}$. Christine Chow $^{47}$. Linda E. Kelemen ${ }^{48}$. Gary L. Keeney ${ }^{49}$. Michael E. Carney ${ }^{50}$. Yanina Natanzon ${ }^{22} \cdot$ Gregory Robertson $^{1,51} \cdot$ Raghwa Sharma $^{52,53} \cdot$ Simon A. Gayther $^{54,55,56} \cdot$ Jennifer Alsop ${ }^{57}$. Hugh Luk $^{9} \cdot$ Chloe Karpinskyj $^{58} \cdot$ lan Campbell ${ }^{59,60} \cdot$ Peter Sinn $^{15} \cdot$ Aleksandra Gentry-Maharaj $^{58} \cdot$ Penny Coulson $^{26}$. Jenny Chang-Claude ${ }^{41,61} \cdot$ Mitul Shah $^{57} \cdot$ Martin Widschwendter ${ }^{58} \cdot$ Katrina Tang $^{17} \cdot$ Minouk J. Schoemaker $^{18}$. Jennifer M. Koziak ${ }^{62}$ • Linda S. Cook ${ }^{63,64}$ - James D. Brenton ${ }^{65}$. Frances Daley ${ }^{66,67}$ • Björg Kristjansdottir ${ }^{34,35}$. Constantina Mateoiu ${ }^{68}$ - Melissa C. Larson 21 - Paul R. Harnett ${ }^{31,69}$ - Audrey Jung ${ }^{41}$ - Anna deFazio ${ }^{31,32}$. Kylie L. Gorringe ${ }^{59}$ - Paul D. P. Pharoah ${ }^{27,57} \cdot$ Parham Minoo $^{4} \cdot$ Colin Stewart $^{40} \cdot$ Oliver F. Bathe $^{70} \cdot$ Xianyong Gui $^{4}$. Paul Cohen $\mathbb{1}^{16,28}$. Susan J. Ramus ${ }^{1,3,71} \cdot$ Martin Köbel $\mathbb{1}^{4}$

1 School of Women's and Children's Health, Faculty of Medicine, University of NSW Sydney, Sydney, NSW, Australia

2 Prince of Wales Clinical School. UNSW Sydney, Sydney, NSW, Australia

3 Adult Cancer Program. Lowy Cancer Research Centre, Sydney, Australia

4 Department of Pathology and Laboratory Medicine, University of Calgary, Foothills Medical Center, Calgary, AB, Canada

5 Pathology Department, Catholic University of Health and Allied Sciences-Bugando, Mwanza, Tanzania

6 British Columbia's Ovarian Cancer Research (OVCARE) Program, Vancouver General Hospital, BC Cancer Agency and University of British Columbia, Vancouver, BC, Canada

7 Department of Molecular Oncology, BC Cancer Agency Research Centre, Vancouver, BC, Canada

8 Department of Pathology and Laboratory Medicine, University of British Columbia, Vancouver, BC, Canada

9 Cancer Epidemiology Program, University of Hawaii Cancer Center, Honolulu, HI, USA

10 Department of Histopathology, Imperial College London, Hammersmith Hospital, London, UK

11 University of Texas MD Anderson Cancer Center, Houston, TX, USA

12 Department of Gynecologic Oncology, Roswell Park Cancer Institute, Buffalo, NY, USA

13 Department of Obstetrics and Gynecology, Division of Gynecologic Oncology. Royal Alexandra Hospital, Edmonton, AB, Canada

14 Tissue Bank of the National Center for Tumor Diseases, University of Heidelberg, Heidelberg, Germany

15 Department of Pathology, Institute of Pathology, University Hospital Heidelberg, Heidelberg, Germany

16 Bendat Family Comprehensive Cancer Centre, St John of God Subiaco Hospital, Subiaco, Western Australia, Australia

17 NSW Health Pathology. Prince of Wales Hospital, Sydney, NSW, Australia

18 Division of Genetics and Epidemiology, The Institute of Cancer Research, London, UK

19 Division of Breast Cancer Research, The Institute of Cancer Research, London, UK
20 Department of Genetics and Pathology, Pomeranian Medical University, Szczecin, Poland

21 Department of Health Science Research, Division of Biomedical Statistics and Informatics. Mayo Clinic, Rochester, MN, USA

22 Department of Health Science Research, Division of Epidemiology. Mayo Clinic, Rochester, MN, USA

23 MRC Clinical Trials Unit at UCL, Institute of Clinical Trials \& Methodology, University College London, London, UK

24 International Hereditary Cancer Center, Department of Genetics and Pathology, Pomeranian Medical University, Szczecin, Poland

25 Division of Cancer Epidemiology and Genetics. National Cancer Institute, Bethesda, MD, USA

26 Division of Genetics and Epidemiology, Institute of Cancer Research, London, UK

27 Centre for Cancer Genetic Epidemiology, Department of Public Health and Primary Care, University of Cambridge, Cambridge, UK

28 Division of Obstetrics and Gynaecology, Faculty of Health and Medical Sciences, University of Western Australia, Crawley, Western Australia, Australia

29 Western Women's Pathology, Western Diagnostic Pathology, Wembley, Western Australia, Australia

30 Prince of Wales Private Hospital, Randwick, NSW, Australia

31 Centre for Cancer Research, The Westmead Institute for Medical Research, The University of Sydney, Sydney, NSW, Australia

32 Department of Gynaecological Oncology, Westmead Hospital, Sydney, NSW, Australia

33 Department of Histopathology. Addenbrookes Hospital, Cambridge, UK

34 Department of Obstetrics and Gynecology, Sahlgrenska Cancer Center, Inst Clinical Scienses, Sahlgrenska Academy at University of Gothenburg, Gothenburg, Sweden

35 Department of Gynecology, Region Västra Götaland, Sahlgrenska University Hospital, Gothenburg, Sweden

36 Division of Cancer Prevention and Control, Roswell Park Cancer Institute, Buffalo, NY, USA

37 Department of Gynecological Surgery and Gynecological Oncology of Adults and Adolescents, Pomeranian Medical University, Szczecin, Poland 
38 Cancer Prevention and Control, Samuel Oschin Comprehensive Cancer Institute, Cedars-Sinai Medical Center, Los Angeles, CA, USA

39 Community and Population Health Research Institute, Department of Biomedical Sciences, Cedars-Sinai Medical Center, Los Angeles, CA, USA

40 Histopathology Department, King Edward Memorial Hospital, Perth, Western Australia, Australia

41 Division of Cancer Epidemiology, German Cancer Research Center (DKFZ), Heidelberg, Germany

42 Department of Obstetrics and Gynecology, Fryderyk Chopin University Hospital No 1, Faculty of Medicine, Rzeszów University, Rzeszów, Poland

43 Ovarian Cancer Center of Excellence, Womens Cancer Research Program, Magee-Womens Research Institute and University of Pittsburgh Cancer Institute, Pittsburgh, PA, USA

44 Division of Gynecologic Oncology, Department of Obstetrics, Gynecology and Reproductive Sciences, University of Pittsburgh School of Medicine, Pittsburgh, PA, USA

45 Womens Cancer Research Center, Magee-Womens Research Institute and Hillman Cancer Center, Pittsburgh, PA, USA

46 University of Hawaii Cancer Center, Honolulu, HI, USA

47 Genetic Pathology Evaluation Centre, Vancouver General Hospital and University of British Columbia, Vancouver, BC, Canada

48 Department of Public Health Sciences, Medical University of South Carolina, Charleston, SC, USA

49 Department of Laboratory Medicine and Pathology, Division of Anatomic Pathology. Mayo Clinic, Rochester, MN, USA

50 Department of Obstetrics and Gynecology, John A. Burns School of Medicine, University of Hawaii, Honolulu, HI, USA

51 St George Private Hospital, Kogarah, NSW, Australia

52 Pathology West ICPMR Westmead, Westmead Hospital, The University of Sydney, Sydney, NSW, Australia

53 University of Western Sydney at Westmead Hospital, Westmead, NSW, Australia

54 Department of Preventive Medicine, Keck School of Medicine, University of Southern California, Los Angeles, CA, USA
55 Center for Cancer Prevention and Translational Genomics, Samuel Oschin Comprehensive Cancer Institute, Cedars-Sinai Medical Center, Los Angeles, CA, USA

56 Department of Biomedical Sciences, Cedars-Sinai Medical Center, Los Angeles, CA, USA

57 Centre for Cancer Genetic Epidemiology, Department of Oncology, University of Cambridge, Cambridge, UK

58 Gynaecological Cancer Research Centre, Women's Cancer, Institute for Women's Health, University College London, London, UK

59 Peter MacCallum Cancer Center, Melbourne, Victoria, Australia

60 Sir Peter MacCallum Department of Oncology, The University of Melbourne, Melbourne, Victoria, Australia

61 Cancer Epidemiology Group, University Cancer Center Hamburg (UCCH). University Medical Center Hamburg-Eppendorf, Hamburg, Germany

62 Alberta Health Services-Cancer Care, Calgary, AB, Canada

63 University of New Mexico Health Sciences Center. University of New Mexico, Albuquerque, NM, USA

64 Department of Cancer Epidemiology and Prevention Research, Alberta Health Services, Calgary, AB, Canada

65 Cancer Research UK Cambridge Institute, University of Cambridge, Cambridge, UK

66 Division of Breast Cancer Research. Institute of Cancer Research, London, UK

67 Division of Bioscience, Brunel University, London, UK

68 Department of Pathology, Sahlgrenska Academy at University of Gothenburg, Gothenburg, Sweden

69 The Crown Princess Mary Cancer Centre Westmead, SydneyWest Cancer Network. Westmead Hospital, Sydney, NSW, Australia

70 Departments of Surgery and Oncology, Tom Baker Cancer Centre, Calgary, AB, Canada

71 The Kinghorn Cancer Centre, Garvan Institute of Medical Research, Sydney, NSW, Australia 